\title{
Polydopamine Induced Wettability Switching of Cellulose Nanofibers/n-Dodecanethiol Composite Aerogels
}

\author{
Runan Gao $\mathbb{D}$, ${ }^{1}$ Ying Shang $\mathbb{D}^{1},{ }^{1}$ Peng Jiao $\mathbb{D},{ }^{1}$ Yue Jiao $\mathbb{D},{ }^{2}$ Jian $\operatorname{Li} \mathbb{D},{ }^{1}$ and Yun Lu $\mathbb{D}^{3}$ \\ ${ }^{1}$ Key Laboratory of Bio-Based Material Science and Technology Ministry of Education, Materials Science and Engineering College, \\ Northeast Forestry University, Harbin 150040, China \\ ${ }^{2}$ College of Materials Science and Engineering, Nanjing Forestry University, Nanjing 210037, China \\ ${ }^{3}$ Research Institute of Wood Industry, Chinese Academy of Forestry, Beijing 100091, China
}

Correspondence should be addressed to Jian Li; jianlinefu@126.com and Yun Lu; y.lu@caf.ac.cn

Received 4 June 2021; Revised 7 November 2021; Accepted 4 December 2021; Published 11 January 2022

Academic Editor: Antonio Caggiano

Copyright (c) 2022 Runan Gao et al. This is an open access article distributed under the Creative Commons Attribution License, which permits unrestricted use, distribution, and reproduction in any medium, provided the original work is properly cited.

\begin{abstract}
The novel wettability switchable cellulose nanofiber- (CNF-) based aerogel was conveniently prepared by polydopamine mediated composition of CNF and n-dodecanethiol. The wettability of aerogels can be controlled by adjusting the PDA and ndodecanethiol loading content, which leads to a variation of water contact angle from $0-149^{\circ}$. The PDA was coated on cellulose nanofibers via hydrogen bonds and then $\mathrm{n}$-dodecanethiol was anchored onto the scaffolds by Michael addition reaction, which was revealed by XPS and FTIR spectra. The composite aerogel can selectively absorb a series of oily liquids from the oil/water mixture, with the maximum absorption capacity of $68 \mathrm{~g} / \mathrm{g}$. This work presented a facile strategy to prepare wettability switchable CNF-based heterogenous aerogel and exhibited the potential of the composite aerogel for oil/water separation.
\end{abstract}

\section{Introduction}

Water pollutant caused by oily liquid spillage is an ongoing problem which is of great urgency to solve worldwide [1, 2]. Common remediation strategies include biological treatment, coagulation-flocculation, in situ burning, and physical oil/water separation. Among them, physical collection/separation with absorbents or filters excels in high efficiency, secondary pollution-free, and potential for oil reclamation [3-8]. Synthetic porous materials and carbon-based sponge have been extensively investigated for oily wastewater remediation $[9,10]$. Delicate surface modification endows them with special wettability and thus selective oil/water separation performance. However, these materials suffer from complicated synthesis, nondegradable composition posing environmental problems.

CNF aerogel has received widely attention due to its sustainability, biocompatibility, and degradability [11-14]. In addition, unique properties including high porosity, large specific surface, and rich in oxygen-containing groups [15-18] make CNF aerogel a promising material for water remediation $[19,20]$. However, when dealing with oily contamination, the intrinsic hydrophilicity of cellulose hindered its use in selective oil/water separation. Many efforts have been devoted to switch the wettability of CNF aerogels. For instance, plasma etching [21, 22] and atomic layer deposition [7] techniques have been reported to create hydrophobic CNF surface. Recently, the chemical vapor deposition and aqueous silylation strategies are widely adapted to alter the wettability of CNF aerogels $[23,24]$. However, most of them suffer from high-cost fabrication procedure and toxic chemicals. Therefore, to develop a facile and universal wettability switching strategy is important for fabricating potential scalable CNF-based oil/water separation materials.

Polydopamine (PDA), a biomimetic polymer of adhesive protein in mussel, has received great attention due to its universal adhesion ability. Polydopamine is capable of coating on virtually all kinds of surface. Functional groups on PDA such as catechol, amine, and imine make it popular for secondary modification. PDA has been extensively coated on organic/inorganic substrates for fabricating superwetting materials, adsorbent materials, photothermal materials, 


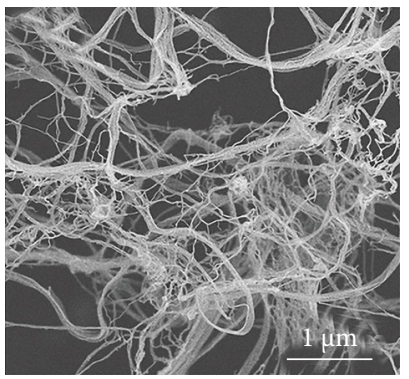

(a)

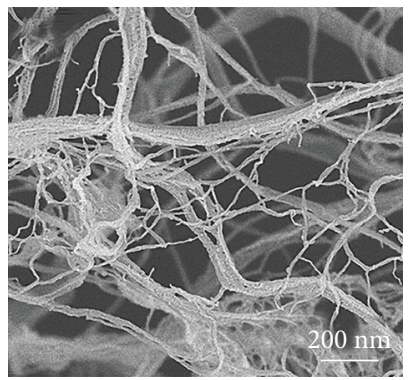

(e)

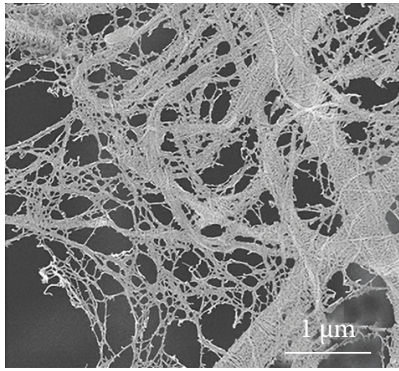

(b)

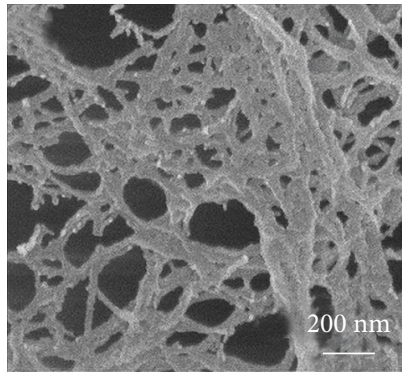

(f)

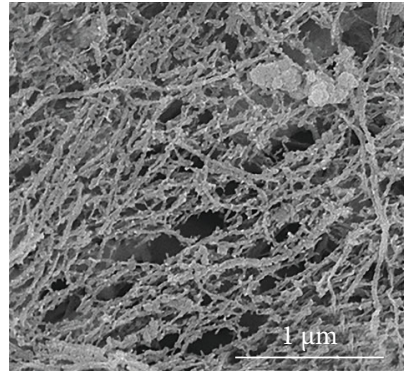

(c)

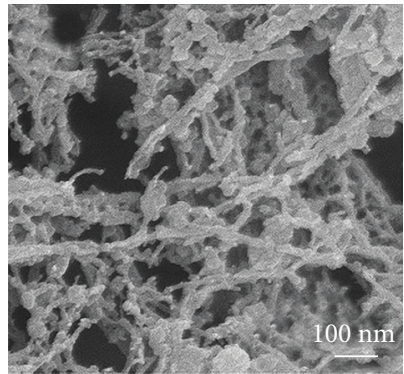

(g)

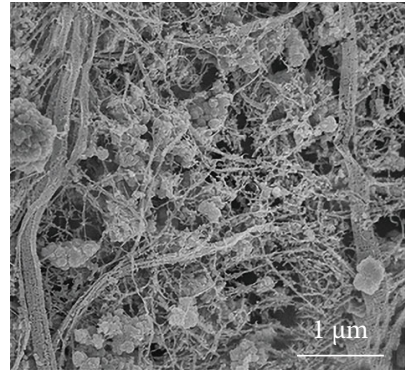

(d)

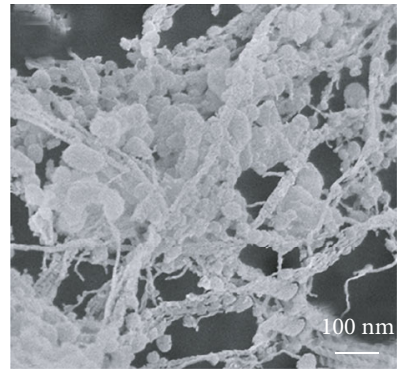

(h)

FIGURE 1: (a)-(d) SEM images of 0.2PDA@CNF, 0.5PDA@CNF, 1PDA@CNF, and 2PDA@CNF, respectively; (e)-(h) corresponding highmagnification images of (a)-(d).

catalytic materials, and so on. For instance, Zheng et al. coated PDA magnetic $\mathrm{Fe}_{3} \mathrm{O}_{4}$ nanoparticles to form $\mathrm{PDA}-\mathrm{Fe}_{3} \mathrm{O}_{4}$ coreshell structure with enhanced near-infrared irradiation absorption, and thus, an enhanced photothermal effect. Shao et al. used PDA coating as the "bio-glue" to bridge polyvinylidene fluoride (PVDF) membrane and $\mathrm{TiO}_{2}$ particles to fabricate ultrafiltration membranes $[25,26,27]$.

Recently, PDA surface chemistry has been widely applied to cellulose-based materials including natural fiber [28-30], cellulose nanocrystals [31,32], electrospun cellulose nanyrans [33-35], and CNF [36-38] to facilely fabricating functional materials. Here, we report a facile approach to prepared hydrophobic CNF aerogels. Simply by one-step reaction followed by freeze-drying, composite aerogel with high water contact angle (WCA) of $149^{\circ}$ was obtained. PDA was coated on CNF and worked as the anchor to bridge hydrophobic n-dodecanethiol (DDT) molecules. The effect of PDA and DDT loading concentration on wettability was investigated, and the composition mechanism between heterogenous molecules was discussed in this paper.

\section{Experiments}

Pine needles of Larix gmelini were collected on the campus of Northeast Forestry University. Dopamine hydrochloride (DA), tris-(hydroxymethyl)-aminomethane (Tris), tris(hydroxymethyl)-aminomethane hydrochloride (Tris- $\mathrm{HCl}$ ), n-Dodecanethiol (DDT) was purchased from Aladdin reagent.

CNF was prepared as previously reported method [39]. Add $1.77 \mathrm{~g}$ Tris- $\mathrm{HCl}$ and $3.5 \mathrm{~g}$ Tris to $400 \mathrm{~mL}$ CNF suspension $(0.1 \mathrm{wt} \%)$ to prepare CNF-Tris solution $(\mathrm{pH}=8.5)$. Divide the CNF-Tris solution $(400 \mathrm{~mL})$ equally into 4 beakers, add 20, 50, 100, and $200 \mathrm{mg}$ of DA, respectively, and react for $24 \mathrm{~h}$ at room temperature. The product was washed with distilled water until $\mathrm{pH}=7$. Dilute the solution with the concentration of $0.05 \mathrm{wt} \%$. Diluted product was freeze-dried at $-50^{\circ} \mathrm{C}$ for $48 \mathrm{~h}$ to obtain PDA@CNF composite framework. According to the dosage of DA, the samples were recorded as 0.2PDA@CNF, 0.5PDA@CNF, 1PDA@CNF, and2PDA@CNF. Obtained samples were kept in desiccator for SEM investigation.

For preparing hydrophobic aerogels, likewise, different PDA@CNF suspensions were prepared first, then $10 \mathrm{~mL}$ ethanol containing $1 \mathrm{~mL}$ DDT was dispersed in different PDA@CNF solutions, magnetically stir for $48 \mathrm{~h}$, after which resulting samples were washed with distilled water for three times. After removing the excess liquid, the samples were poured into molds and freeze-dried at $-50^{\circ} \mathrm{C}$ for $48 \mathrm{~h}$ to obtain the DDT-PDA@CNF aerogels. According to the different dosage of DA, the materials are denoted as DDT0.2PDA@CNF, DDT-0.5PDA@CNF, DDT-1PDA@CNF, and DDT-2PDA@CNF.

\section{Characterization}

Morphology of the samples was characterized with Scanning Hitachi JSM-7500F scanning electron microscope (SEM). Fourier transform infrared spectroscopy (FTIR) data was recorded with Nicolet Nexus 670, scanning range is 4000$600 \mathrm{~cm}^{-1}$. X-ray photoelectron spectroscopy (XPS) was used to characterize the structural changes of the sample before and after the interface functionalization. Water contact angle (WCA) was recorded with Data Physics OCA 20 contact angle measuring instrument. A TA Q600 thermal gravimetric analyzer (TGA) was used to analyze the thermal stability of the samples, test range: room temperature to $800^{\circ} \mathrm{C}$, heat-

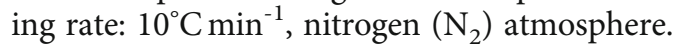




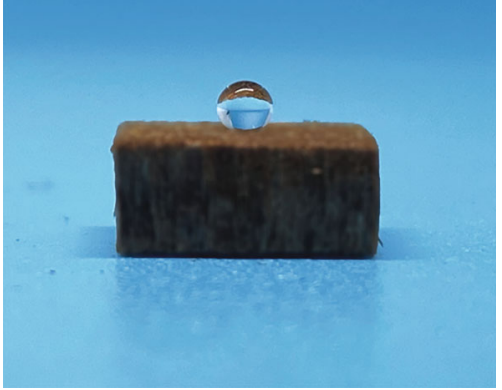

(a)

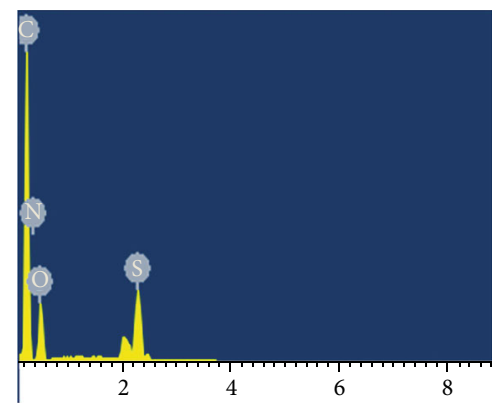

(d)

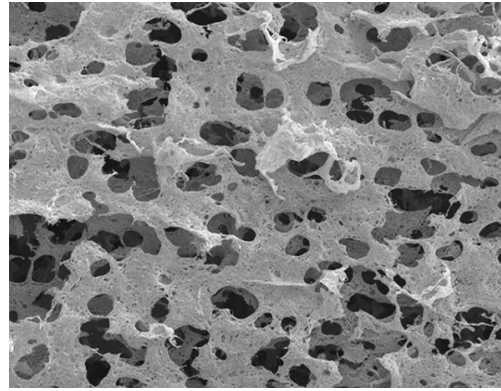

(b)

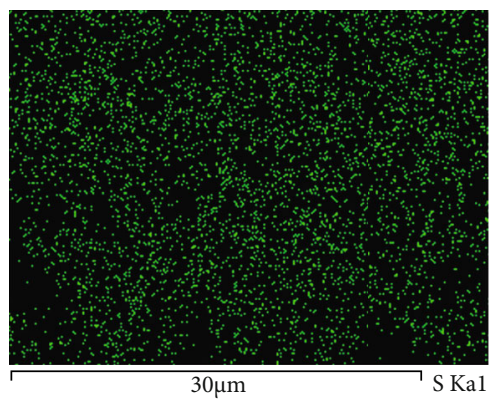

(e)

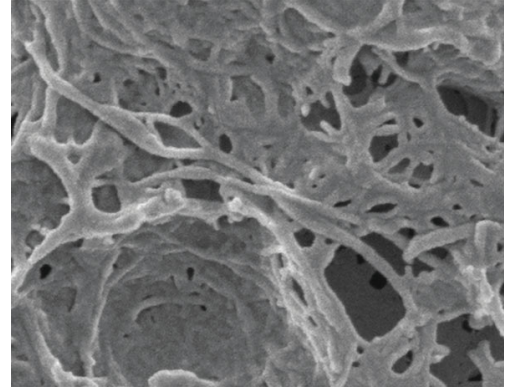

(c)

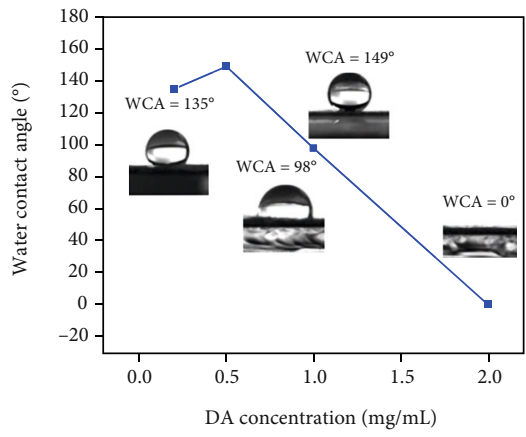

(f)

Figure 2: (a) Photo of DDT-0.5PDA@CNF aerogel, (b) and (c) SEM images of DDT-0.5PDA@CNF, (d) and (e) EDS and S element mapping image of DDT-0.5PDA@CNF, and (f) WCA of composite aerogels with different DA concentration.

TABle 1: The mean diameter of different PDA@CNF composite scaffolds.

\begin{tabular}{lc}
\hline Sample & Mean diameter $(\mathrm{nm})$ \\
\hline $0.2 \mathrm{PDA} @ \mathrm{CNF}$ & 28 \\
$0.5 \mathrm{PDA} @ \mathrm{CNF}$ & 34 \\
1PDA@CNF & 39 \\
2PDA@CNF & 41 \\
\hline
\end{tabular}

\section{Results and Discussion}

The morphology of PDA@CNF scaffolds with different DA concentration was investigated by SEM. Figures 1(a)-1(d) show the low-magnification SEM pictures of 0.2PDA@CNF, 0.5PDA@CNF,1PDA@CNF, and 2PDA@CNF, respectively. Figures $1(\mathrm{e})-1(\mathrm{~h})$ are the high-magnification SEM pictures corresponding to Figures $1(\mathrm{a})-1(\mathrm{~d})$. The initial concentration of CNF in all samples was $1 \mathrm{mg} / \mathrm{mL}$. As shown in Figure 1(a), the morphology of 0.2PDA@CNF is similar to that of pure CNF, which are entangled with each other demonstrating a three-dimensional network structure. A thin layer of gravel-like particles was evenly attached to the surface of the CNF, as shown in Figure 2(e), indicating that the PDA coating has been loaded onto the CNF surface. When the concentration of DA increases to $0.5 \mathrm{mg} / \mathrm{mL}$, the PDA coating fully covers CNF scaffolds, as revealed in Figures 1(b) and 1(f). Different from the independently dispersed fibers in 0.2PDA@CNF,0.5PDA@CNF adheres to each other, which makes the one-dimensional composite

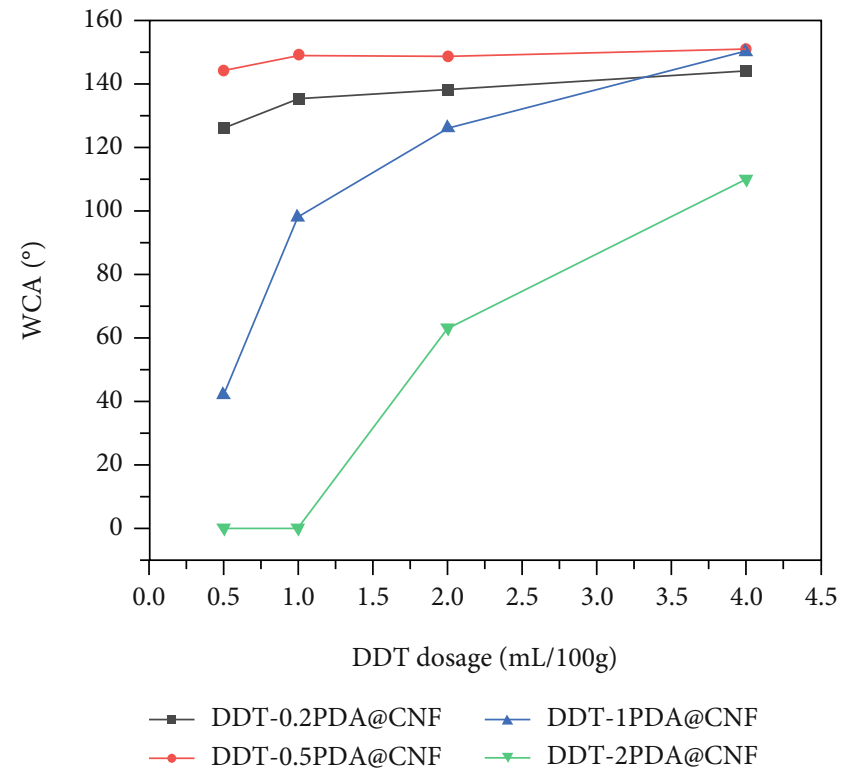

FIgURE 3: The WCA of composite aerogels with different DDT loading content.

fibers transform into to two-dimensional flakes. This may be due to the adhesive characteristics of PDA. When the dosage of DA increases to $1 \mathrm{mg} / \mathrm{mL}$, the PDA coating on $\mathrm{CNF}$ is further thickened, and spherical PDA particles appear on the composite surface, as shown in Figures 1(c) and $1(\mathrm{~g})$. PDA particles are evenly distributed on the composite scaffolds. When the concentration of DA increases 


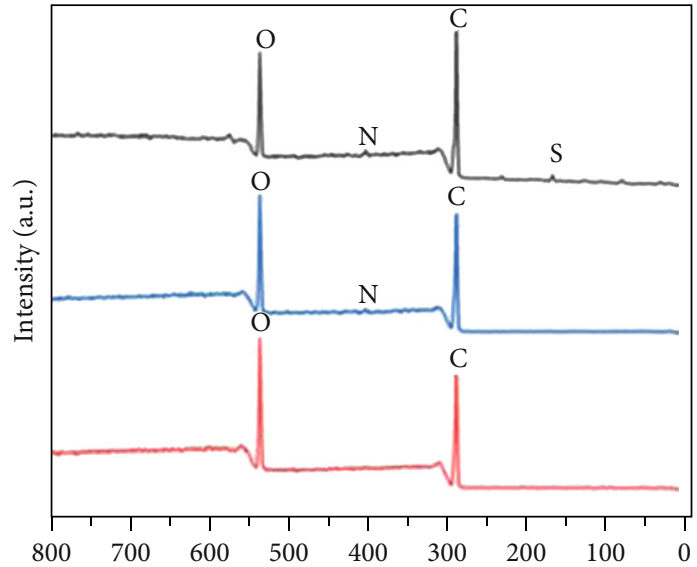

Binging energy (eV)

(a)

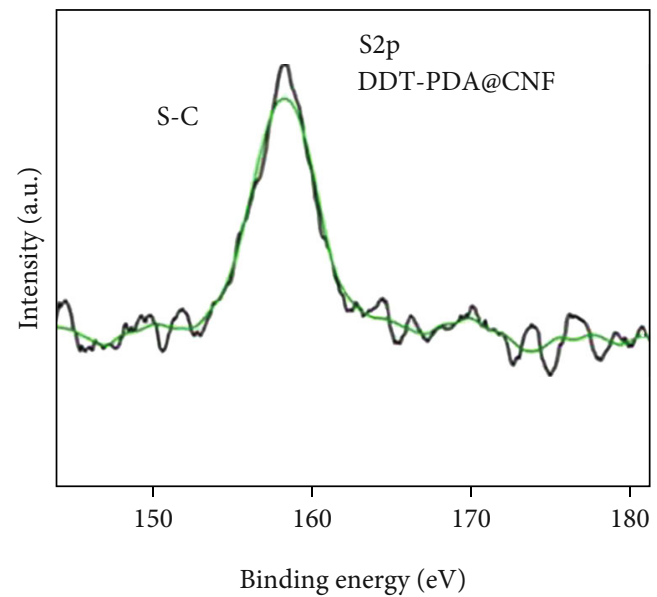

(c)

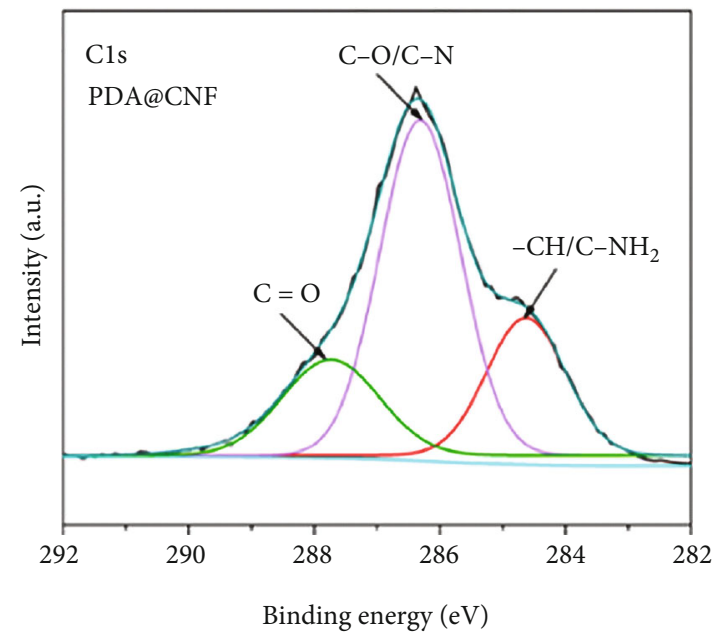

(e)

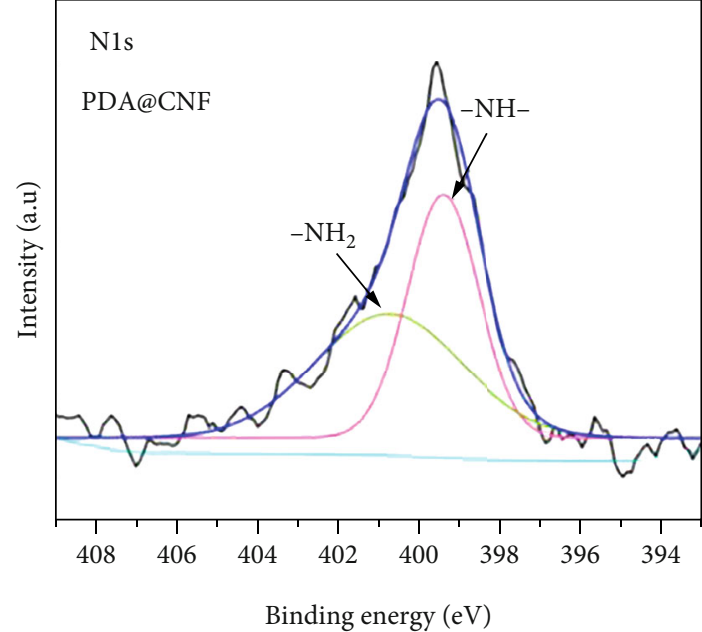

(b)

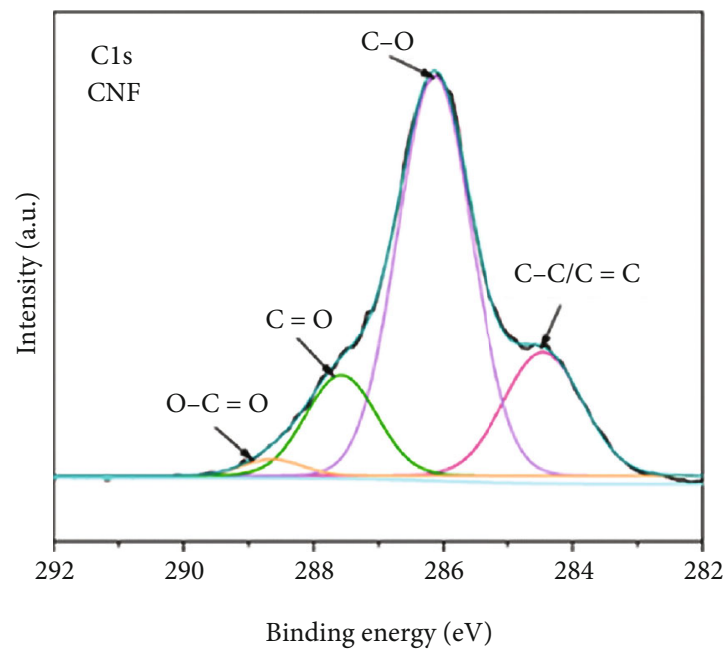

(d)

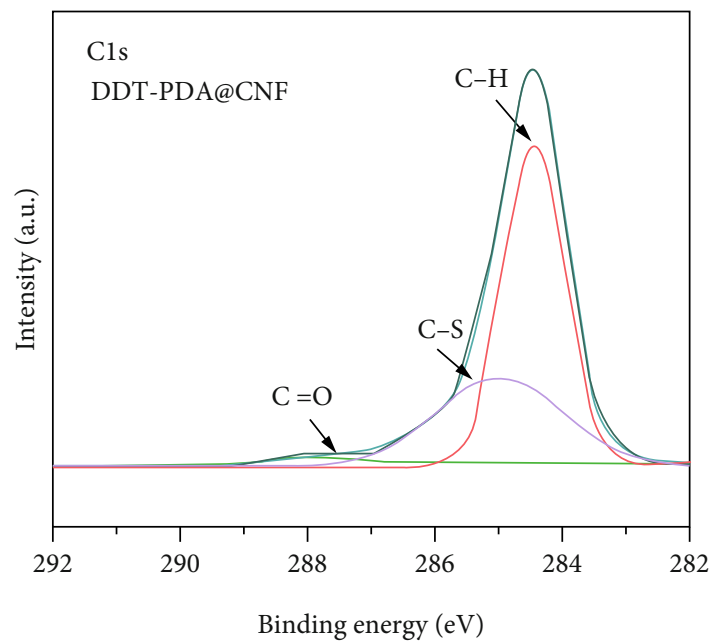

(f)

FIGURE 4: (a) Down to top: XPS survey spectrum of CNF, PDA@CNF, and DDT-PDA@CNF, (b) high resolution N 1s spectrum of PDA@CNF, (c) high resolution S 2p spectrum of DDT-0.5PDA@CNF, and (d)-(f) high resolution C $1 \mathrm{~s}$ spectrum of CNF, 0.5PDA@CNF, and DDT-0.5PDA@CNF. 


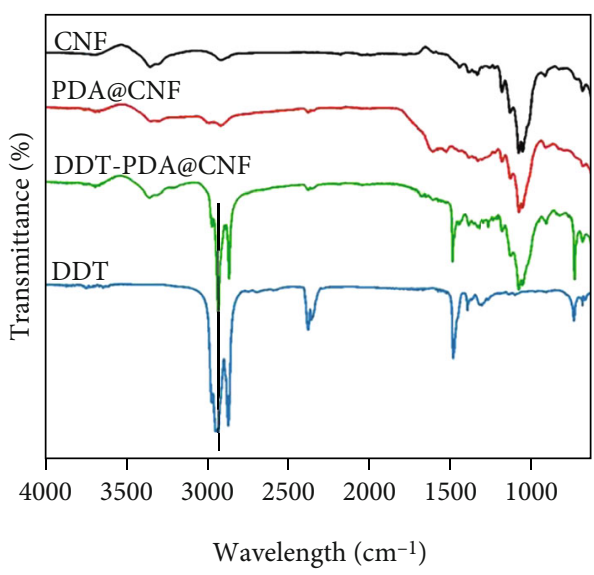

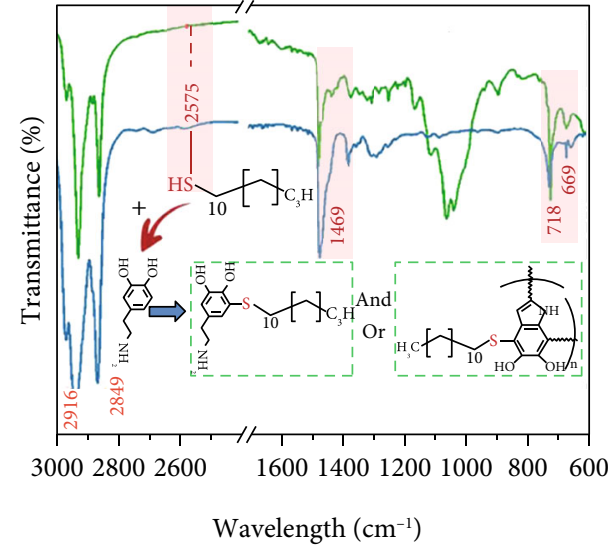

— DDT-PDA@CNF

— Pure DDT

FIGURE 5: (a) FTIR spectrum of CNF, 0.5PDA@CNF, and DDT-0.5PDA@CNF and (b) zoom-in FTIR spectrum of DDT-0.5PDA@CNF and pure DDT in range of 3000 to $600 \mathrm{~cm}^{-1}$.

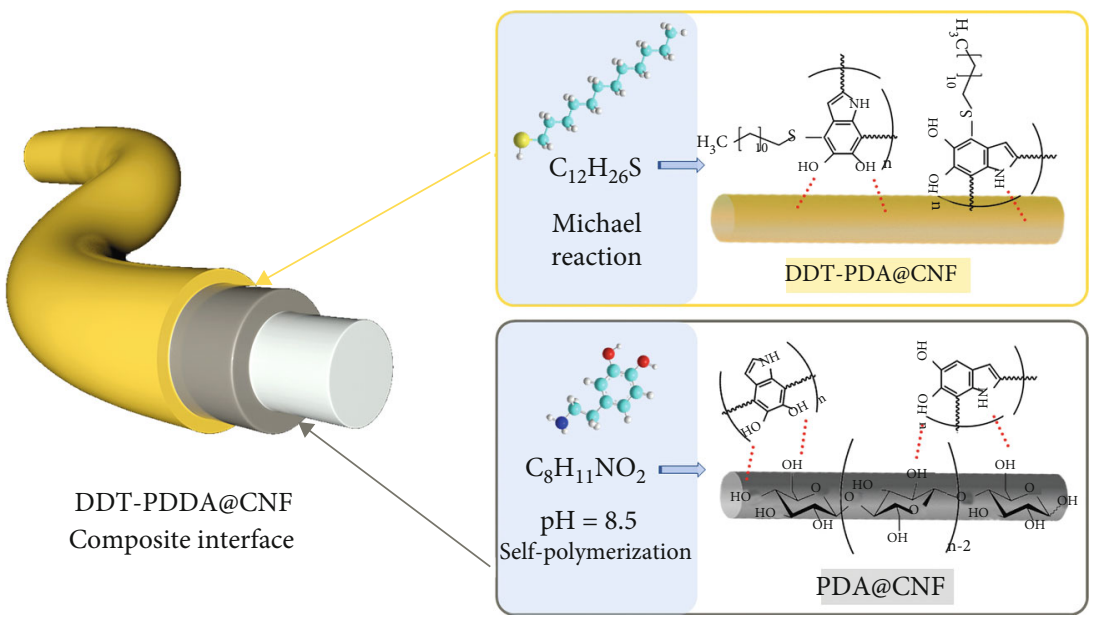

FIGURE 6: The graphic illustration of constructing the DDT-PDA@CNF composite interface.

TABLE 2: Bulk densities and porosities and specific surface areas of $\mathrm{CNF}$ and composite aerogels.

\begin{tabular}{lccc}
\hline Sample & $\begin{array}{c}\text { Density } \\
\left(\mathrm{mg} / \mathrm{cm}^{3}\right)\end{array}$ & Porosity $(\%)$ & $\begin{array}{c}\text { Specific surface } \\
\text { area }\left(\mathrm{m}^{2} / \mathrm{g}\right)\end{array}$ \\
\hline CNF & 2.03 & 99.96 & 177 \\
0.2PDA@CNF & 2.41 & 99.8 & 171.3 \\
0.5PDA@CNF & 3.07 & 99.23 & 154.6 \\
1PDA@CNF & 4.42 & 98.61 & 129.3 \\
2PDA@CNF & 5.13 & 98.14 & 77.1 \\
DDT-0.5PDA@CNF & 4.82 & 99.07 & 79.4 \\
\hline
\end{tabular}

to $2 \mathrm{mg} / \mathrm{mL}$, the morphology of the 2PDA@CNF composite framework does not change significantly, while the amount and the diameter of PDA microspheres are significantly increased, as revealed in Figures 1(d) and 1(f). Some spheres are gathered into clusters and fixed in composite skeleton to block the pores. As the DA dosage increases from 0.2 to
$2 \mathrm{mg} / \mathrm{mL}$, the mean diameter of composite scaffolds expands from 28 to $41 \mathrm{~nm}$, as shown in Table 1 .

Results from SEM images indicate that the DA solution with a concentration of $0.5 \mathrm{mg} / \mathrm{mL}$ is sufficient to induce a uniform coating on CNF surface. In addition, when the DA concentration increases to $1 \mathrm{mg} / \mathrm{mL}$, spherical PDA particles appear and attach to networks.

As shown in Figure 2(a), after introducing DDT onto 0.5PDA@CNF, the resulting aerogel shows hydrophobicity with WCA of 149. The composite scaffolds were assembled to three-dimensional (3D) porous structure during freezedrying (Figure 2(b)). Figure 2(c) is the high-magnification SEM image of DDT-0.5PDA@CNF, the wax-like substance wrapped on the surface of DDT-0.5PDA@CNF. As mentioned before, when the DA dosage is $0.5 \mathrm{mg} / \mathrm{mL}$, a uniform PDA coating is formed on the CNF surface, as a result, DDT was oriented and evenly covered on 0.5PDA@CNF.

EDS scanning and $\mathrm{S}$ element mapping were performed as shown in Figures $2(\mathrm{~d})$ and $2(\mathrm{c})$. In addition to $\mathrm{C}$ and $\mathrm{O}$ 

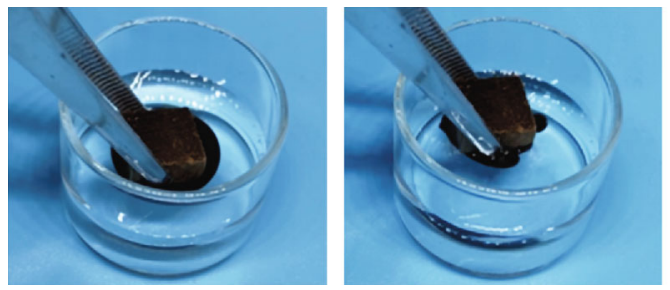

(a)
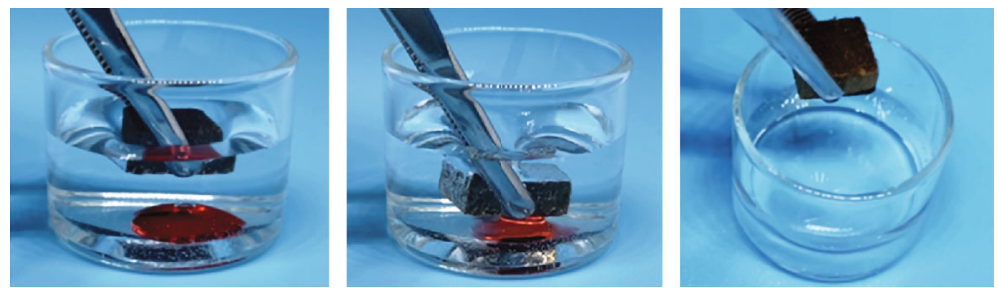

(b)

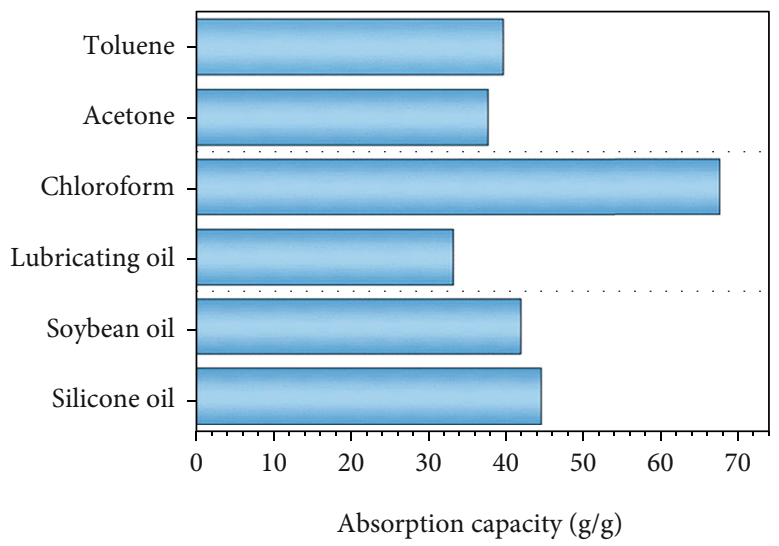

(c)

FIGURE 7: Absorption capacity of DDT-0.5PDA@CNF toward some oily liquids (a), separation the dyed toluene (b) and (c) chloroform from the water phase with DDT-0.5PDA@CNF.

elements, nitrogen $(\mathrm{N})$ elements in PDA and sulfur (S) elements in DDT appeared on the surface of aerogel. It can be observed in Figure 2(d) that the S element is widely distributed on the surface of the material. The above results preliminarily indicate that DDT has been loaded on the surface of $0.5 \mathrm{PDA} @ \mathrm{CNF}$, forming hydrophobic DDT-0.5PDA@CNF composite material.

Figure 2(f) reveals the wettability of composite aerogels with different DA dosage. DA and its self-polymerized product PDA are intrinsically hydrophilic. Thus, it is important to investigate the effect of DA concentration on WCA of final composite aerogels. The WCA of composite aerogels increases from $135^{\circ}$ to $149^{\circ}$ with the DA dosage increasing from 0.2 to $0.5 \mathrm{mg} / \mathrm{mL}$, then it sharply decreases to $0^{\circ}$ as the DA dosage increasing to $2 \mathrm{mg} / \mathrm{mL}$. As revealed before, the DA dosage of $0.5 \mathrm{mg} / \mathrm{mL}$ is sufficient to form a uniform PDA coating on CNF. The PDA coating works as anchor which enables DDT evenly linked to composite scaffolds and forms hydrophobic surface. However, when the concentration of DA is more than $1.0 \mathrm{mg} / \mathrm{mL}$, the WCA of DDT0.5PDA@CNF has a sharp decrease.

The DDT loading capacity also has an impact on the wettability of the composite aerogel, as shown in Figure 3.
The WCA of composite aerogels is positively correlated with the DDT loading content, especially for DDT-1PDA@CNF and DDT-2PDA@CNF. When the DDT dosage is increased from $0.5 \mathrm{~mL}$ to $2 \mathrm{~mL}$, the WCA was increased from $43^{\circ}$ to $149^{\circ}$ for DDT-1PDA@CNF and from 0 to $110^{\circ}$ for DDT2PDA@CNF, respectively. While for DDT-0.5PDA@CNF, the dosage of DDT has a slight effect on the WCA, which indicates that the DDT-0.5PDA@CNF has reached the loading saturation with $1 \mathrm{~mL}$ DDT.

XPS and FTIR investigation were used to analyze the chemical evolution of the samples. Figure 4(a) shows the XPS survey spectrum of CNF, 0.5PDA@CNF, and DDT0.5PDA@CNF, respectively. After modification, the signal of N element appears in both spectra of 0.5PDA@CNF and DDT-0.5PDA@CNF scaffolds. Take a deep insight into deconvolved high resolution N $1 \mathrm{~s}$ spectra (Figure 4(b)), the peaks at 401.2 and $399 \mathrm{eV}$ are correspondence to $-\mathrm{NH}_{2}$ and -NH- moieties of the PDA, respectively, which proves that the PDA coating contains the dopamine dimer and indole group structure. After introducing DDT, the signal of S element appears in XPS survey spectrum indicating DDT has been successfully coated on the composite skeleton. Figure $4(\mathrm{c})$ is the high-resolution XPS spectrum of S 
2p. It is worth noting that peaks at $162 \mathrm{eV}$ correspond to C-S bond rather than H-S bond. This shows that the sulfhydryl group $(-\mathrm{SH})$ at the ending moiety of the DDT was consumed during the reaction, which indicates DDT molecules were grafted to PDA@CNF scaffolds via Michael reaction. In Figure 4(d), peaks at 284.6, 286.7, 288.3, and $289.2 \mathrm{eV}$ correspond to the $\mathrm{C}-\mathrm{C}, \mathrm{C}-\mathrm{O}, \mathrm{C}=\mathrm{O}$, and $\mathrm{O}-\mathrm{C}=\mathrm{O}$ bonds in cellulose, respectively. As shown in Figure 4(e), the characteristic peaks at 284.5, 285.8, and 287.7 eV correspond to $-\mathrm{CH} / \mathrm{C}-\mathrm{NH}_{2}, \mathrm{C}-\mathrm{O} /$ $\mathrm{C}-\mathrm{N}$, and $\mathrm{C}=\mathrm{O}$ bonds, respectively. In the $\mathrm{C} 1 \mathrm{~s}$ spectrum of DDT-0.5PDA@CNF, a characteristic peak of C-S bond appears at $286.5 \mathrm{eV}$, which further indicates that DDT is loaded on the surface of composite scaffolds.

Figure 5(a) shows the FTIR spectra of CNF, 0.5PDA@CNF, and DDT-0.5PDA@CNF. The broad peak at $3600-3000 \mathrm{~cm}^{-1}$ originates from the stretching vibration of the $\mathrm{H}$-bonded hydroxyl $(-\mathrm{OH})$ in cellulose. The peak at $2900 \mathrm{~cm}^{-1}$ is assigned to $\mathrm{CH}$ stretching vibration, peaks at $1428 \mathrm{~cm}^{-1}$ and $1370 \mathrm{~cm}^{-1}$ are assigned to $\mathrm{CH}_{2}$ wagging and bending, and the peak at $1050 \mathrm{~cm}^{-1}$ is the in-plane antisymmetric stretching vibration of the $\mathrm{C}-\mathrm{O}-\mathrm{C}$ in pyranose ring of cellulose. After coating the PDA on CNF surface, the peak around $3300 \mathrm{~cm}^{-1}$ was broadened, indicating that functional groups on PDA formed new hydrogen bonding with CNF, as shown in Figure 5. In addition, the new peak at $1508 \mathrm{~cm}^{-1}$ is the characteristic peak assigned to N-H on the PDA coating. So far, it can be proved that PDA was successfully coated onto the CNF surface.

Figure 5(b) is the partial FTIR spectrum of pure DDT and DDT-0.5PDA@CNF. Peaks at 2916 and $2849 \mathrm{~cm}^{-1}$ indicate the antisymmetric and symmetry stretching vibration of $\left(-\mathrm{CH}_{2}-\right)$ in DDT molecules. The characteristic peak of -SH at $2575 \mathrm{~cm}^{-1}$ disappears in the FITR spectrum of DDT0.5PDA@CNF, indicating most of the sulfhydryl groups are consumed during reaction process. Peaks at 718 and $669 \mathrm{~cm}^{-1}$ are characteristic signal of C-S bond in the thiol ligand, which are significantly enhanced in the FTIR spectrum of DDT-0.5PDA@CNF, which indicates that during the composite constructing process, the mercaptan groups in thiol are gradually consumed, and graft to the aromatic ring in PDA via Michael reaction and formed new C-S bond, which further proved that DDT is covalent bonded to PDA@CNF scaffolds, as revealed in Figure 6.

The bulk density, porosity, and specific surface area (SSA) of CNF and composites aerogels are listed in Table 2. As the PDA and DDT are coated on the CNF, the bulk density is gradually increased, while the porosity and SSA gradually decreased.

As revealed in Figure 7, taking the toluene and the chloroform as examples, the DDT-0.5PDA@CNF aerogel can rapidly separate organic solvents from water. The hydrophobic coating enables the composite aerogel to be absorbed oily liquids but block the water. Absorption performance of DDT-0.5PDA@CNF for some oily liquids was investigated. The mass-based absorption capacity, to a large extent, depends on the solvent density. The absorption capacities of DDT-0.5PDA@CNF for different oil and organic solvents are 33.6 to $68.1 \mathrm{~g} / \mathrm{g}$, which is comparable some polyurethane sponges $(25-87 \mathrm{~g} / \mathrm{g})$.

\section{Conclusions}

In summary, a wettability switchable CNF-based aerogels by compositing with PDA and DDT molecules was reported in this work. Polydopamine works both as the capitalizer to bridge heterogenous cellulose/n-dodecanethiol molecules as well as the controller to switch the wettability of the composite aerogels. FTIR and XPS results show that PDA was first coated on CNF by hydrogen bonding, then, DDT was anchored on the composited scaffold by Michael reaction. DDT-0.5PDA@CNF aerogel showed a maximum WCA of $149^{\circ}$, and it can fast absorb a series of oily liquids with the maximum absorption capacity reach up to $68 \mathrm{~g} / \mathrm{g}$.

\section{Data Availability}

The FTIR and XPS data used to support the findings of this study are included within the article.

\section{Conflicts of Interest}

The authors declare that they have no conflicts of interest.

\section{Authors' Contributions}

Runan Gao and Yun Lu conceived the idea for this study. Li Jian supervised the work. Runan Gao designed experiments and wrote the manuscript. Peng Jiao prepared composite aerogels and test the absorption performance. Yue Jiao performed characterization of samples. Ying Shang and Peng Jiao contributed equally to this work.

\section{Acknowledgments}

This study was supported by the Key Laboratory of Biobased Material Science and Technology (Northeast Forestry University), Ministry of Education, and the National Natural Foundation of China (grant no. 31870535).

\section{References}

[1] Y. Zou, J. Zhao, J. Zhu et al., "A mussel-inspired polydopamine-filled cellulose aerogel for solar-enabled water remediation," ACS Applied Materials \& Interfaces, vol. 13, no. 6, pp. 7617-7624, 2021.

[2] J. Jiang, J. Zhu, Q. Zhang, X. Zhan, and F. Chen, "A shape recovery zwitterionic bacterial cellulose aerogel with superior performances for water remediation," Langmuir, vol. 35, no. 37, pp. 11959-11967, 2019.

[3] D. A. Gopakumar, D. Pasquini, M. A. Henrique, L. C. de Morais, Y. Grohens, and S. Thomas, "Meldrum's acid modified cellulose nanofiber-based polyvinylidene fluoride microfiltration membrane for dye water treatment and nanoparticle removal," ACS Sustainable Chemistry \& Engineering, vol. 5, no. 2, pp. 2026-2033, 2017.

[4] G. J. Vadakkekara, S. Thomas, and C. P. R. Nair, "Sodium itaconate grafted nanocellulose for facile elimination of lead ion from water," Cellulose, vol. 27, no. 6, pp. 3233-3248, 2020.

[5] B. D. Deshpande, P. S. Agrawal, M. K. N. Yenkie, and S. J. Dhoble, "Prospective of nanotechnology in degradation of 
waste water: a new challenges," Nano-Structures \& NanoObjects, vol. 22, article 100442, 2020.

[6] L. Yi, J. Yang, X. Fang et al., "Facile fabrication of woodinspired aerogel from chitosan for efficient removal of oil from water," Journal of Hazardous Materials, vol. 385, article 121507, 2020.

[7] X. Zhao, X. Wang, and T. Lou, "Preparation of fibrous chitosan/sodium alginate composite foams for the adsorption of cationic and anionic dyes," Journal of Hazardous Materials, vol. 403, article 124054, 2021.

[8] N. Bagheri, M. Mansour Lakouraj, V. Hasantabar, and M. Mohseni, "Biodegradable macro-porous CMC-polyaniline hydrogel: synthesis, characterization and study of microbial elimination and sorption capacity of dyes from waste water," Journal of Hazardous Materials, vol. 403, article 123631, 2021.

[9] Y. Liu, X. Wang, and S. Feng, "Nonflammable and magnetic sponge decorated with polydimethylsiloxane brush for multitasking and highly efficient oil-water separation," Advanced Functional Materials, vol. 29, no. 29, article 1902488, 2019.

[10] W. Lyu, J. Li, L. Zheng et al., "Fabrication of 3D compressible polyaniline/cellulose nanofiber aerogel for highly efficient removal of organic pollutants and its environmental-friendly regeneration by peroxydisulfate process," Chemical Engineering Journal, vol. 414, article 128931, 2021.

[11] T. K. Ghosh, S. Sadhukhan, D. Rana et al., "Chemically reduced graphene oxide (CRGO) from waste batteries and morphological assessment of $\mathrm{CRGO} /$ methyl cellulose transdermal film," Nano-Structures \& Nano-Objects, vol. 22, article 100454, 2020.

[12] J. R. Nair, F. Bella, N. Angulakshmi, A. M. Stephan, and C. Gerbaldi, "Nanocellulose-laden composite polymer electrolytes for high performing lithium-sulphur batteries," Energy Storage Materials, vol. 3, pp. 69-76, 2016.

[13] W. Gan, Y. Wang, S. Xiao et al., "Magnetically driven 3D cellulose film for improved energy efficiency in solar evaporation," ACS Applied Materials \& Interfaces, vol. 13, no. 6, pp. 7756-7765, 2021.

[14] M. Jaroszewski, S. Thomas, and A. V. Rane, Advanced Materials for Electromagnetic Shielding: Fundamentals, Properties and Applications, John Wiley \& Sons, Inc., USA, 2019.

[15] D. A. Gopakumar, A. R. Pai, Y. B. Pottathara et al., "Cellulose nanofiber-based polyaniline flexible papers as sustainable microwave absorbers in the X-band," ACS Applied Materials \& Interfaces, vol. 10, no. 23, pp. 20032-20043, 2018.

[16] D. A. Gopakumar, A. R. Pai, Y. B. Pottathara et al., "Flexible papers derived from polypyrrole deposited cellulose nanofibers for enhanced electromagnetic interference shielding in gigahertz frequencies," Journal of Applied Polymer Science, vol. 138, no. 16, article 50262, 2021.

[17] A. R. Pai, T. Binumol, D. A. Gopakumar et al., "Ultra-fast heat dissipating aerogels derived from polyaniline anchored cellulose nanofibers as sustainable microwave absorbers," Carbohydrate Polymers, vol. 246, article 116663, 2020.

[18] A. R. Pai, C. Paoloni, and S. Thomas, "Chapter 10 nanocellulose-based sustainable microwave absorbers to stifle electromagnetic pollution," in Nanocellulose Based Composites for Electronics, S. Thomas and Y. B. Pottathara, Eds., pp. 237258, Elsevier, 2021.

[19] Y. Lu, H. Liu, R. Gao et al., "Coherent-interface-assembled Ag2O-anchored nanofibrillated cellulose porous aerogels for radioactive iodine capture," ACS Applied Materials \& Interfaces, vol. 8, no. 42, pp. 29179-29185, 2016.

[20] R. Gao, S. Xiao, W. Gan et al., "Mussel adhesive-inspired design of superhydrophobic nanofibrillated cellulose aerogels for oil/water separation," ACS Sustainable Chemistry \& Engineering, vol. 6, no. 7, pp. 9047-9055, 2018.

[21] R. Lin, A. Li, T. Zheng, L. Lu, and Y. Cao, "Hydrophobic and flexible cellulose aerogel as an efficient, green and reusable oil sorbent," RSC Advances, vol. 5, no. 100, pp. 82027-82033, 2015.

[22] X. Zhou, Y. Liu, C. Du et al., "Free-standing sandwich-type graphene/nanocellulose/silicon laminar anode for flexible rechargeable lithium ion batteries," ACS Applied Materials \& Interfaces, vol. 10, no. 35, pp. 29638-29646, 2018.

[23] X. Zhang, M. Liu, H. Wang, N. Yan, Z. Cai, and Y. Yu, "Ultralight, hydrophobic, anisotropic bamboo-derived cellulose nanofibrils aerogels with excellent shape recovery via freezecasting," Carbohydrate Polymers, vol. 208, pp. 232-240, 2019.

[24] H. Zhang, J. Wang, G. Xu, Y. Xu, F. Wang, and H. Shen, "Ultralight, hydrophobic, sustainable, cost-effective and floating kapok/microfibrillated cellulose aerogels as speedy and recyclable oil superabsorbents," Journal of Hazardous Materials, vol. 406, article 124758, 2021.

[25] P. Samyn, "Polydopamine and cellulose: two biomaterials with excellent compatibility and applicability," Polymer Reviews, vol. 61, no. 4, pp. 814-865, 2021.

[26] J. H. Ryu, P. B. Messersmith, and H. Lee, "Polydopamine surface chemistry: a decade of discovery," ACS Applied Materials \& Interfaces, vol. 10, no. 9, pp. 7523-7540, 2018.

[27] Y. Liu, K. Ai, and L. Lu, "Polydopamine and its derivative materials: synthesis and promising applications in energy, environmental, and biomedical fields," Chemical Reviews, vol. 114, no. 9, pp. 5057-5115, 2014.

[28] A. Bourmaud, J. Riviere, A. Le Duigou, G. Raj, and C. Baley, "Investigations of the use of a mussel-inspired compatibilizer to improve the matrix-fiber adhesion of a biocomposite," Polymer Testing, vol. 28, no. 6, pp. 668-672, 2009.

[29] M. Zhou, Y. Li, C. He, T. Jin, K. Wang, and Q. Fu, "Interfacial crystallization enhanced interfacial interaction of poly (butylene succinate)/ramie fiber biocomposites using dopamine as a modifier," Composites Science and Technology, vol. 91, pp. 22-29, 2014.

[30] M. Zhou, J. Yan, Y. Li et al., "Interfacial strength and mechanical properties of biocomposites based on ramie fibers and poly(butylene succinate)," RSC Advances, vol. 3, no. 48, pp. 26418-26426, 2013.

[31] S. Liu, Y. Chen, C. Liu, L. Gan, X. Ma, and J. Huang, "Polydopamine-coated cellulose nanocrystals as an active ingredient in poly(vinyl alcohol) films towards intensifying packaging application potential," Cellulose, vol. 26, no. 18, pp. 9599-9612, 2019.

[32] L. Li, R.-Y. Bao, T. Gao et al., "Dopamine-induced functionalization of cellulose nanocrystals with polyethylene glycol towards poly ( ${ }_{\mathrm{L}}$-lactic acid) bionanocomposites for green packaging," Carbohydrate Polymers, vol. 203, pp. 275-284, 2019.

[33] Z. Yang, J. Si, Z. Cui et al., "Biomimetic composite scaffolds based on surface modification of polydopamine on electrospun poly(lactic acid)/cellulose nanofibrils," Carbohydrate Polymers, vol. 174, pp. 750-759, 2017.

[34] X. Song, Y. Zhang, Y. Wang, M. Huang, S. Gul, and H. Jiang, "Nanocomposite membranes embedded with dopamine- 
melanin nanospheres for enhanced interfacial compatibility and nanofiltration performance," Separation and Purification Technology, vol. 242, article 116816, 2020.

[35] T. Shih, N. Liu, Q. Zhang et al., "Preparation of DOPA-TA coated novel membrane for multifunctional water decontamination," Separation and Purification Technology, vol. 194, pp. 135-140, 2018.

[36] A. D. Dwivedi, N. D. Sanandiya, J. P. Singh et al., "Tuning and characterizing nanocellulose interface for enhanced removal of dual-sorbate (AsVand CrVI) from water matrices," ACS Sustainable Chemistry \& Engineering, vol. 5, no. 1, pp. 518-528, 2017.

[37] Z. Wang, S. Zhao, W. Zhang, C. Qi, S. Zhang, and J. Li, "Bioinspired cellulose nanofiber-reinforced soy protein resin adhesives with dopamine-induced codeposition of "water-resistant" interphases," Applied Surface Science, vol. 478, pp. 441-450, 2019.

[38] R. Hollertz, V. L. Durán, P. A. Larsson, and L. Wågberg, "Chemically modified cellulose micro- and nanofibrils as paper-strength additives," Cellulose, vol. 24, no. 9, pp. 38833899, 2017.

[39] S. Xiao, R. Gao, Y. Lu, J. Li, and Q. Sun, "Fabrication and characterization of nanofibrillated cellulose and its aerogels from natural pine needles," Carbohydrate Polymers, vol. 119, pp. 202-209, 2015. 